\title{
Heterogeneidad en las microempresas informales mexicanas: evidencia empírica y algunas implicaciones de política
}

\section{René Rivera Huerta*}

Perfiles Latinoamericanos, 26(51)

2018 | pp. 63-87

DOI: $10.18504 / \mathrm{pl} 2651-003-2018$

\begin{abstract}
Resumen
A diferencia de las teorías tradicionales del desarrollo económico, nuevas corrientes consideran que las microempresas informales en actividades no agropecuarias son un sector dinámico de la economía. No obstante, unas y otras reconocen la necesidad de diseñar políticas destinadas a formalizar estas empresas e incrementar su productividad. Usando datos de microempresas mexicanas para el ańo 2008 y técnicas de análisis clúster, este artículo propone que, dada la alta heterogeneidad entre las microempresas informales, las políticas a aplicar deben ser distintas. Así, mientras ciertas empresas se beneficiarían de la creación de políticas productivas, otras podrían requerir políticas de asistencia social.
\end{abstract}

\begin{abstract}
Unlike traditional theories of development, new schools of thinking consider nonfarm informal micro-enterprises as a dynamic sector. Nevertheless, social researchers from both streams recognize the necessity of policies to formalize and increase the productivity of such kind of enterprises. Using Mexican data from 2008 and cluster analysis techniques, this work proposes that informal micro-enterprises constitute a very heterogeneous group and that such heterogeneity deserves a diversified strategy of development: while some entrepreneurs would benefit from productivity policies, some others would require an assistance approach.
\end{abstract}

Palabras clave: sector informal, desarrollo inclusivo, microempresas, estrategia de desarrollo, análisis clúster, México.

Keywords: Informal sector, inclusive development, micro-enterprises, strategy of development, cluster analysis, Mexico.

* Doctor en Economía por la Scuola Superiore Sant'Anna. Profesor investigador en la Universidad Autónoma Metropolitana, Unidad Xochimilco | rivera.uam@gmail.com 


\section{Introducción ${ }^{1}$}

$\mathrm{D}$ iversas contribuciones académicas han concebido tradicionalmente al sector informal como un obstáculo para el desarrollo de los sistemas económicos y sociales y, por tanto, se le refiere de continuo como un refugio de los trabajadores que han sido incapaces de encontrar un espacio en la economía formal (Fields, 2004, 2005). En consecuencia, las políticas que se desprenden de dichas contribuciones se orientan, casi en exclusiva, a la reducción/desaparición del sector informal formalizándolo mediante diversos mecanismos. ${ }^{2}$

No obstante, una perspectiva más reciente de las políticas dirigidas al sector informal vive un radical cambio. Así, dentro del estudio del proceso de desarrollo, una fracción de la academia ha otorgado un cada vez más importante papel al "corazón" del sector informal: las microempresas informales ${ }^{3}$ en actividades no agropecuarias. En efecto, de acuerdo a esta corriente, este tipo de empresas ha mostrado el suficiente dinamismo para constituirse, desde el punto de vista de los trabajadores, en una alternativa viable al empleo asalariado formal y para contribuir, en algunos casos, al sostenimiento y el desarrollo de los sistemas económicos, en particular de los países más pobres (Adams et al., 2013). Este nuevo paradigma arguye que las políticas dirigidas a solucionar el problema que la informalidad implica son, por necesidad, complejas y deben ser dependientes del contexto. Además, no excluyen el reconocimiento de las organizaciones informales, aceptando que algunas actividades en pequeña escala deben recibir apoyo a pesar de su falta de formalidad (Chen et al., 2001; Chen, 2007; Cozzens \& Sutz, 2014).

Se observa entonces una discrepancia en las políticas sugeridas por diversas corrientes de la academia para solucionar los problemas que acompañan a las

1 Este artículo es parte del proyecto de investigación "Explorando los mecanismos de aprendizaje e innovación en la microempresa informal" llevado a cabo en la Universidad Autónoma Metropolitana y financiado por el Conacyt (número de proyecto 168379). Agradezco a Alejandra Mendoza y Alena Juárez por su gentil asistencia durante el desarrollo de este texto.

2 El sector informal está constituido, de acuerdo a muchos autores (Gërxhani, 2004: p. 274; Tokman, 2011), por las empresas que carecen de registro o licencia ante una dependencia de gobierno (Castell \& Portes, 1989; Swaminathan, 1991). Por tanto, dentro del sector informal es posible encontrar dos tipos de trabajadores: los asalariados y los propietarios de microempresas; estos últimos incluyen tanto a empleadores como a trabajadores por cuenta propia (INEGI, 2004; Tokman, 2011; Chen, 2012).

3 Este trabajo utiliza una definición operativa de microempresa informal muy similar a la del Instituto Nacional de Estadística y Geografía (INEGI), el cual considera como uno de los criterios fundamentales para clasificar una empresa como informal que sea de tamaño micro (INEGI, 2004: p.19); por eso, en lo que resta del artículo, concebir un negocio en este sector remite a una microempresa. Cabe mencionar que la definición del INEGI se basa en las normas internacionales actuales sobre el empleo en el sector informal adoptadas por la 15a Conferencia Internacional de Estadísticos del Trabajo (CIET) de 1993, y en las directrices adoptadas en la $17^{\mathrm{a}}$ CIET de 2003 (oIT, 2013). 
empresas informales; es posible que la misma tenga su causa en la diferencia de los fundamentos con que dichas corrientes teóricas se sostienen y a la heterogeneidad de las microempresas informales (Fields, 2004; Nguimkeu, 2013), la cual impide focalizar el problema en estudio. Así, una parte de la literatura intuye que entre las empresas informales es posible encontrar actividades de "supervivencia" y otras propiamente empresariales u "orientadas al crecimiento" que comparativamente pueden ser admitidas como exitosas. En este sentido, cabría preguntarse: ¿la evidencia empírica soporta la percepción de heterogeneidad entre las microempresas informales? De ser afirmativa la respuesta, ¡Cuáles características diferencian las microempresas informales "orientadas al crecimiento" de aquellas de "supervivencia"? Presentar algunas respuestas es un primer objetivo de esta investigación.

Una segunda pregunta-objetivo del artículo se formularía de la siguiente forma: ¿̨la dicotomía "crecimiento-supervivencia" es suficiente para explicar la vasta heterogeneidad hallada entre las empresas informales? Aun cuando esta clasificación ha resultado de utilidad en la discusión teórica, simplificar de más podría frenar la gestación de políticas apropiadas a las microempresas con estatus de informalidad y su aplicación.

Finalmente, una vez aceptada la heterogeneidad de los negocios informales, es razonable suponer que no todas las estrategias de políticas son las mejores para influir positivamente en la situación de los empresarios que pertenecen a las dos categorías señaladas ni para optimizar el desarrollo colectivo. Por ello, y con base en las diferencias de nuestra clasificación, ¿las actuales estrategias aplicadas por el gobierno mexicano son las apropiadas?

Para responder, en este artículo se utilizan técnicas clasificatorias de análisis clúster en dos etapas y se usan datos oficiales de México recopilados por el Instituto Nacional de Estadística, Geografía e Informática (INEGI) en la Encuesta Nacional de Micronegocios (ENAmin) correspondientes al año 2008, los cuales proporcionan información de las características de empresa y empresario, es decir, que solo contienen información a nivel micro. Se espera que los conglomerados resultantes permitan diferenciar las actividades de las microempresas informales de acuerdo con su nivel de productividad y así asociarlas con los rasgos de su propietario. Esta contribución es entonces un primer esfuerzo hacia la creación de una tipología de esta clase de negocios y se tiene la expectativa de que los resultados promuevan la discusión sobre el tema y coadyuven en la formulación de políticas dirigidas - en un contexto mexicano- a cada agrupación de las mismas.

El artículo se estructura de la siguiente forma: después de presentar las diferencias entre las propuestas de políticas provenientes de las principales teorías ortodoxas que han abordado el tema de la informalidad y las derivadas de lo que algunos autores consideran una nueva visión del tema, se procede a revisar 
brevemente la literatura acerca de la heterogeneidad entre las microempresas informales. Enseguida se establece el método seguido para clasificar las empresas y se describen la base de datos y las variables utilizadas. Por último, se exponen los resultados del análisis clúster y el estudio de estos mismos, esbozando una primera propuesta de política orientada a las empresas con estatus de informalidad.

\section{Diferentes corrientes teóricas; diferentes propuestas de política}

A pesar de la gran cantidad de publicaciones de los últimos años en torno al tema, la naturaleza del sector informal sigue siendo poco clara y tan controversial como en sus inicios. En forma temprana, cuatro teorías se enfocaron en entender el origen y la naturaleza de este sector. Así, mientras la teoría dualista lo considera como de supervivencia (Fields, 2004, 2005), la propuesta contraria, denominada a veces voluntarista (Chen, 2012), lo califica de competitivo e incluso de superior a su contraparte formal (Maloney, 1999, 2004). Por otra parte, corrientes teóricas menos extremas, como la legalista, afirman que el sector informal es consecuencia de un sistema institucional que reprime el espíritu empresarial de los más pobres dentro de los países en proceso de desarrollo (Soto, 1989). Finalmente, otra escuela de pensamiento lo observa como unidades productivas al servicio de las empresas del sector formal las cuales, con la contratación de empresas informales, buscan obtener mayor flexibilidad y competitividad en un mundo globalizado (Castells \& Portes, 1989; Tokman, 1995). ${ }^{4}$

Las principales teorías que han abordado el fenómeno social y económico del sector informal difieren entre sí al explicar su origen y la dinámica de su reproducción. Por esa discrepancia sería factible suponer que sus propuestas de solución serían distintas entre sí, sin embargo, todas coinciden en observar con pesimismo, o cuando menos con escepticismo, el papel del sector informal en el desarrollo económico de las sociedades. Adicionalmente, ninguna propuesta de política derivada de las corrientes teóricas citadas ha sido realmente exitosa al momento de explicar la evidencia empírica y, en consecuencia, las políticas que de ellas se deriven deben ser reconsideradas con cuidado (Rivera-Huerta, 2014).

Es debido a la persistencia del sector informal que algunos autores han generado la llamada nueva visión con propuestas de políticas dirigidas a apoyar las actividades de dicho sector y aprovechar así sus contribuciones en la economía y en la sociedad (Kumar \& Bhaduri, 2014; Tokman, 2001; Chen et al., 2001).

4 Desde diversas perspectivas, en la literatura se registran revisiones atinadas de la naturaleza, del concepto y de la definición operativa del sector informal, véanse Tokman (1995), Gërxhani (2004), Chowdury (2005) y Coletto (2010: pp. 1-42). 
En esta línea, diversos trabajos académicos han evidenciado que las actividades informales —en particular las de las empresas - no son necesariamente improductivas ${ }^{5}$ (Nguimkeu, 2013; Chen et al., 2001). Es seguro que, debido a alguna evidencia similar, autores relacionados con organismos internacionales como la Organización Internacional del Trabajo (оIт) (Bangasser, 2000) y, en fechas más recientes, organismos de perfil más académico como Women in Informal Employment: Globalizing and Organizing (wIEGo) (Chen et al., 2001) y el International Development Research Centre (IDRC) (Cozzens \& Sutz, 2014) han propuesto la creación de políticas de desarrollo más amigables con las actividades informales. Esas políticas van desde el microfinanciamiento y la vinculación con el sector formal hasta el fomento y el impulso del aprendizaje, la capacitación y la innovación.

\section{Heterogeneidad entre las microempresas informales}

Como ya se afirmó arriba, entre las propuestas de políticas dirigidas a mejorar las condiciones de los trabajadores (propietarios de empresas y empleados) que operan las microempresas con estatus de informalidad existe discrepancia. Esta diferencia de percepción respecto de lo que se requiere para mitigar los efectos negativos de la informalidad es probable que se encuentre asociada a las bases teóricas subyacentes en las corrientes académicas enfocadas a estudiar la naturaleza y dinámica de las empresas informales, pero también, sin duda, a la heterogeneidad en dicho sector y a la parte que de este han observado los investigadores.

Es de tiempo atrás que se considera que la diversidad es una característica intrínseca del sector informal y que en este coexisten actividades clasificadas como superiores (upper-tier) ${ }^{6}$ con otras inferiores (lower-tier) (Fields, 2005). En el caso específico de las microempresas informales, la heterogeneidad ha sido, hasta don-

5 Se ha sugerido que el sector informal no tiene un efecto negativo obligado en la economía y que, en algunos aspectos, puede resultar positivo tanto para los países desarrollados como para aquellos en desarrollo (Schneider, 1998; Adams et al., 2013). Quizá el mejor ejemplo de que una política dirigida a soportar las actividades informales puede resultar exitosa lo brinda el experimento natural de la Emilia-Romagna (Capecchi, 1989), una región ubicada en el centro-norte de Italia, cuyo actual alto índice de desarrollo se fundamenta en un origen de empresas y vínculos informales. Tal desarrollo no se dio solo con las empresas informales, sino en combinación con características idiosincrásicas como una cultura de cooperación y una complicidad de apoyo mutuo entre los productores informales, además del apoyo de un gobierno de izquierda (Capecchi, 1989). Esta conjunción de variables impulsó el desarrollo regional, el cual pasó de la producción agrícola a la de aparatos electrónicos y a generar servicios de alto impacto.

6 Upper tier y lower tier son términos que han llegado a ser populares en la literatura especializada para designar empresas informales de alto desempeńo y bajo desempeño, respectivamente. Por tal causa se usarán estos términos a lo largo del artículo.

R. Rivera Huerta | Heterogeneidad en las microempresas informales mexicanas: evidencia empírica y algunas implicaciones de política | Perfiles Latinoamericanos, 26(51) | Flacso México | pp. 63-87 
de el conocimiento nos permite afirmar, menos tratada y se le ha clasificado en dos amplias categorías relacionadas con el comportamiento de sus propietarios. La primera de ellas es relativamente exitosa y agrupa a las empresas orientadas al crecimiento, es decir, pequeños negocios cuyos propietarios siguen grosso modo el comportamiento del agente maximizador, en línea con las teorías ortodoxas de la economía, distinguiéndose así de las empresas de supervivencia, en las cuales los empresarios buscan seguridad sobre los elementos esenciales de la vida y cuyas actividades muchas veces son desarrolladas ante la falta de oportunidad en otras áreas de trabajo y se caracterizan, entre otros aspectos, por la falta de capital físico o humano. Este es en esencia el concepto usado por Berner et al. (2012), Nguimkeu (2013) y Sinclair-Desgagné (2013).

A pesar de su sencillez, esta separación entre empresas orientadas al crecimiento y las de supervivencia basta para entender que las políticas dirigidas a ellas también deben adaptarse a sus características y, por ende, a las de sus propietarios. La necesidad de políticas diferenciadas ya había sido destacada por Sinclair-Desgagné (2013), quien, a partir de la teoría de la decisión y tomando en cuenta los beneficios hipotéticos de tener un estatus de formalidad, así como de las características psicológicas propias de los empresarios (tolerancia al riesgo, ambición, etc.), construye un modelo matemático para observar las condiciones bajo las cuales un empresario decide o no ser formal. Según dicho autor, los empresarios escogerán el estatus de formalidad versus el de informalidad en la medida en que posean determinados rasgos (optimismo, por ejemplo), encuentren un beneficio en dicho estatus y presupongan que su empresa tiene potencial de crecimiento.

A partir de estos resultados, Sinclair-Desgagné arriesga algunas recomendaciones de política, entre las que propone incentivar la entrada de los empresarios —específicamente de los más exitosos_ a la formalidad. En ese sentido, sería deseable reducir los costos de formalización manteniéndolos, sin embargo, lo suficientemente altos para conservar dentro de la informalidad las empresas menos rentables y prometedoras, a fin de que no compitan por los recursos de capital y humanos, que se consideran escasos. En resumen, lo que Sinclair-Desgagné propone es crear políticas de apoyo (reduciendo impuestos, fortaleciendo las regulaciones del mercado y la propiedad intelectual, entre otros) dirigidas a las empresas con mayor potencial de crecimiento.

Tal propuesta de clasificación es compartida por Berner et al. (2012). Sin embargo, estos autores observan que la proporción de empresarios orientados al crecimiento es mucho menor que los de supervivencia. Debido a diversos factores - entre los que figuran los culturales—, al segundo grupo no le es posible acumular capital y, por eso, si se reflexiona el objetivo, la aplicación de estrategias para fortalecer su crecimiento sería un gasto de recursos poco eficiente. 
Para este tipo de empresas, Berner et al. recomiendan implementar políticas de apoyo para amortiguar la pobreza, así como políticas de largo plazo de empleo, educación y capacitación.

\section{¿Cuáles variables determinan la heterogeneidad en las empresas informales?}

Si se reconoce que las microempresas informales constituyen un conjunto heterogéneo, es indispensable aproximarse a su estructura para comprender la pertinencia de las políticas a aplicar y una primera forma de hacerlo sería entendiendo las características asociadas a cada subgrupo del universo de empresas en informalidad.

Acerca de ello, una primera fuente de información se constituiría de las investigaciones que suponen una categorización entre los microempresarios informales; sin embargo, la literatura sobre este aspecto es más bien escasa. No obstante, una fuente de información adicional y útil resulta ser la abundante literatura que estudia las empresas formales en sus variables de crecimiento, supervivencia y cierre.

Debido al tamaño de las empresas informales, es natural que su desempeño se vincule estrechamente a las características del propietario. De este modo y por su importancia, lo primero a ser considerado tendría que ser el capital humano. Así, la educación formal del microempresario es una de las pocas variables que ha abordado la literatura del tema, y revisándola fue que se identificó un efecto positivo entre ella y un mayor dinamismo (Nguimkeu, 2013; Adams et al., 2013). Otro factor a considerar y que también pertenece al ámbito del capital humano es la experiencia del empresario - bastante relacionado con la edad-y el tipo específico de la misma. Esta variable resulta clave en la gestión de una empresa y quien previamente aprenda a hacerlo mejor obtendrá más éxito (Dahl \& Reichstein, 2007). Por ejemplo, Berner et al. (2012) asocian los empresarios categorizados como orientados al crecimiento con una experiencia previa como asalariados en el sector formal. Mientras que Campbell (1992), citado en Garoma (2012), señala que la importancia de la experiencia radica tanto en el conocimiento adquirido como en los vínculos creados, y Kumar \& Bhaduri (2014) subrayan el peso de este elemento, al afirmar que se trata de la base de la innovación que se da en las empresas informales.

Por otra parte, el nivel de capital y el ingreso constituyen candidatos lógicos como variables determinantes de la heterogeneidad de las empresas informales. $\mathrm{Al}$ respecto, Berner et al. han advertido que los empresarios orientados al crecimiento nunca son pobres por definición (Berner et al., 2012). En tanto que, 
para el caso de Camerún, Nguimkeu (2013) presenta evidencia de que la disposición de capital inicial explica en gran medida la heterogeneidad observada en el retorno de capital de las microempresas informales.

Otras características sociodemográficas que se deben incorporar en el análisis son el género y el estatus migratorio de los propietarios. En cuanto a lo primero, la literatura ha notado una brecha de ingresos entre microempresarios y microempresarias en el sector informal, lo que sucede a tal punto que a la mayor parte de estas últimas se les identifica con las empresas de supervivencia (Berner et al., 2012). Ello se explica parcialmente porque el promedio del nivel de educación y el de experiencia son menores entre las mujeres a causa de que se desempeñan en actividades menos productivas, pero, como Freije (2001) señala, es una cuestión que todavía no está bien investigada. Por otro lado, los primeros estudios (Harris \& Todaro, 1970; Charmes, 1995) asociaban el creciente fenómeno de la informalidad urbana a la migración rural hacia las ciudades impulsado por un diferencial en los ingresos en ambas regiones. Dado el escaso capital físico y humano que en general se atribuye al migrante campesino es factible relacionar esta condición a las actividades de supervivencia.

La edad del empresario asimismo influye en la heterogeneidad del sector informal. Welter (2001), por ejemplo, arguye que, aunque los jóvenes suelen ser más ambiciosos y determinados que los más viejos, estos últimos poseen mayores recursos, con lo que se establece una relación positiva entre la edad y el crecimiento de la empresa. Esto concordaría con lo hipotetizado por Maloney (2004), quien propone que los empleados se tornan informales (trabajadores por cuenta propia o patrones) después de haber adquirido un cierto nivel de capital; el necesario para echar a andar su pequeña empresa. Sin embargo, este investigador en coautoría con Cunningham (Cunningham \& Maloney, 1999) y usando técnicas clúster e información de 1992 para microempresas mexicanas, presentaría evidencia de que, sin educación formal, los individuos con mayor edad percibirían una media de ingresos menor que otras categorías más afortunadas de microempresarios.

Para finalizar en cuanto a las variables relacionadas de forma directa con los empresarios, hay que mencionar que los rasgos psicológicos incrementan fuertemente la probabilidad de que se les asocie con el grupo orientado al crecimiento. De esta forma, Baum et al. (2001) indican que la motivación, la visión y la confianza en sí mismo del empresario juegan un papel fundamental en el crecimiento de la empresa. Mientras que Sinclair-Desgagné, siguiendo a Kirhlstrom \& Laffont (1979), concluye que un mayor optimismo y menor aversión al riesgo hacen mejores empresarios y que, por ende, dichas características podrían llevarlos incluso a la formalización. Un punto de vista compartido por Berner et al. (2012). 
Por otro lado, algunas variables relacionadas con las características propias de las empresas podrían incidir en su categorización. Así, la edad y el tamaño han sido dos de los factores más estudiados. Una empresa con mayor edad ha probado su eficiencia — de otra forma habría salido del mercado-, aunque, según Jovanovic (1982), crece con más lentitud. Esta relación negativa se replica entre tamaño y crecimiento. Ambos resultados han sido corroborados por distintos estudios empíricos como, por ejemplo, el de Goedhuys \& Sleuwaegen (2010). De hecho, el tiempo que duran o sobreviven las pequeñas empresas se vincula directamente con el crecimiento (McPherson, 1995).

Es factible, por otra parte, identificar el tipo de microempresa entendiendo si tiene trabajadores adicionales al propietario (Mead \& Liedholm, 1998). Esta variable conduce a dividir entre empresarios que son patrones o empleadores, y trabajadores por cuenta propia (Nguimkeu, 2013).

Además, al desempeño y dinámica de la microempresa le afecta el sector al que pertenece y su localización. Es por ello que Liedholm (2002) refiere que, para ciertos países de África, actividades de manufactura, como el procesamiento de lana, tienen más posibilidad de supervivencia que el comercio en pequeño. Este mismo autor ha encontrado que las empresas localizadas en áreas urbanas y zonas comerciales presentan mayor probabilidad de éxito que las ubicadas en zonas rurales o las establecidas en la casa del propietario.

Por último, variables relativas al capital social y la interacción con empresas más grandes (formales) y de cooperación con otras informales juegan un papel importante en el desempeño de estas últimas (Van Dijk, 2008; Kriastensen, 2004).

\section{Clasificación de las microempresas. Metodología}

Ya se dijo antes que uno de los objetivos principales de este artículo consiste en explorar empíricamente la combinación de variables correlacionadas con el "éxito" relativo de una empresa, es decir, entender qué variables se asocian a la categoría orientada al crecimiento y cuales a la de supervivencia. Por eso es que este trabajo comparte los propósitos de aportaciones como las de Nguetse (2009) y Nguimkeu (2013). Si bien, al mismo tiempo, se pretende destacar que la simplicidad de la dicotomía crecimiento-supervivencia con la que suele clasificarse a las empresas informales, aunque útil para abstraer el proceso de decisión de los empresarios, genera problemas operativos para dictaminar políticas adecuadas dirigidas a mejorar la situación de los negocios del sector informal y eventualmente formalizarlos; dicha dicotomía ignora que, para efectos prácticos, hay empresas que se ubicarían en un lugar intermedio o diferente a los dos polos de esa clasificación y que requieren, por esto mismo, políticas particulares. Un 
primer paso hacia la identificación de estas empresas (de haberlas) constituye un segundo propósito de este artículo. A continuación, se detalla la metodología que aquí se utiliza para alcanzar los objetivos propuestos.

\section{Técnica de clasificación. El análisis clúster}

El análisis clúster es un conjunto de técnicas multivariantes con las que se agrupa objetos basándose en sus propiedades comunes, es decir, que son idóneos para clasificar objetos. El resultado son agrupamientos o conglomerados internamente homogéneos, pero con alta heterogeneidad externa. Sin embargo, este tipo de análisis tiene diversas limitaciones. Por ejemplo, es esencialmente descriptivo y no inferencial y por ello se utiliza sobre todo para fines exploratorios (el objetivo de este trabajo). Además, las soluciones a las que llega no son únicas y dependen de las variables incluidas en el procedimiento, de este modo, la adición o exclusión de variables relevantes impactan en el resultado. A pesar de tales inconvenientes, es una técnica ampliamente usada en la investigación para examinar la naturaleza de los datos (Hair et al., 1999; Romesburg, 2004).

Las técnicas de análisis clúster se dividen en dos grandes grupos: los procedimientos jerárquicos y los no jerárquicos. Sin embargo, en este trabajo se aplicó un procedimiento de análisis clúster en dos etapas, disponible en sPSS (versión 21.0). Se trata de un procedimiento con todas las características de los jerárquicos y los no jerárquicos, con el beneficio extra de que maneja variables categóricas y continuas, permite la selección automática del número de clústeres y tiene la propiedad de manejar bases de datos grandes. En un primer paso, esta técnica usa un enfoque secuencial para hacer el preagrupamiento de las observaciones. Cada una de estas se fusiona en un subclúster previamente formado o empieza uno nuevo dependiendo de los rasgos estadísticos individuales de las variables utilizadas en la categorización.

El objetivo es calcular una nueva matriz de datos con un número menor de observaciones para la siguiente etapa. Enseguida (agrupamiento de casos), de forma similar a las técnicas jerárquicas de aglomeración, los preclústeres obtenidos en el paso previo se les fusiona hasta que todos los clústeres forman uno solo. El algoritmo también provee un número óptimo de clústeres basados en una combinación de un valor bajo del criterio bayesiano de Schwartz (свS) y una medida alta de la distancia. ${ }^{7}$

7 Una explicación adicional del análisis clúster en dos etapas puede verse en Pérez López (2004) y SPSS Inc. (2001). 
La base de datos que se utilizó fue generada con la Encuesta Nacional de Micronegocios 2008 (ENAMIN), la cual es de carácter mixto y se realizó a partir de la Encuesta Nacional de Ocupación y Empleo (ENOE); esta última es un esfuerzo conjunto del Instituto Nacional de Estadística y Geografía (INEGI) y de la Secretaría del Trabajo y Previsión Social (sTPS) para conocer información estadística de los micronegocios en México y de los trabajadores que la operan. La ENAMIN 2008 tiene representatividad nacional y considera localidades urbanas y rurales de todo tamaño, y contiene registros de 26079 individuos con información; no obstante, en este análisis solo se considera a aquellos que reconocen haber laborado en la semana inmediata anterior a la entrevista.

\section{Definición operativa de microempresa e informalidad}

La población de interés en este estudio se constituye de las microempresas informales en actividades no agropecuarias. Por tanto, es importante definir lo que se entiende aquí como informal y microempresa. Todas las empresas que no cumplieron con los criterios para ser consideradas como microempresas informales fueron excluidas de la muestra.

Microempresa. Para este concepto se ha utilizado la definición del INEGI, el cual determina que una microempresa es una unidad económica de hasta seis trabajadores, incluido el dueño, cuyas actividades pueden darse en cualquier sector, excepto en la manufactura. En el caso específico de las microempresas manufactureras se las concibe como tales con hasta 16 personas, considerado el dueño. Cabe agregar que, en la definición de microempresa de la ENAMIN se contempla también a las empresas con el propietario como único trabajador, es decir, a los empleados por cuenta propia (INEGI, 2009: p. 240; INEGI, 2012: p. 16).

Informalidad. Siguiendo el concepto propuesto de Castell \& Portes (1989), se considerará como informal a todas las microempresas que, a pregunta expresa de la ENAMIN, no reportan su registro en alguna dependencia de gobierno, sea local o federal.

\section{Variables usadas en la categorización}

Las variables para conformar las categorías se relacionan con lo que la literatura especializada reconoce como rasgos de las empresas comprendiendo aquellas que 
se aproximan al desempeño de las pequeñas y micro empresas (véanse las secciones previas del artículo). Dado el tamaño y la naturaleza de las empresas bajo estudio, el perfil del empresario necesariamente afecta la naturaleza de su empresa. Así pues, el análisis que sigue ha incorporado variables asociadas a la empresa y las que caracterizan al empresario.

Características de los empresarios:

- Edad. Variable discreta que indica la edad de los trabajadores expresada en años. Se consideró solo los microempresarios entre 16 y 85 años.

- Experiencia. Variable discreta expresada en años que indica la experiencia laboral del trabajador a partir de la edad en la cual comenzó a trabajar.

- Género. Variable dicotómica binaria que toma el valor de 1 si el empresario es hombre, y 0 si es mujer.

- Experiencia asalariada. Es una variable dicotómica que toma el valor de 1 si el empresario tiene experiencia como trabajador asalariado, y de 0 en caso contrario.

- Migración. Variable dicotómica con valor igual a 1 si el encuestado nació en la ciudad donde tiene su empresa y con valor de 0 si ello no es así.

- Escolaridad. Número de años de educación formal recibida por el trabajador. En la construcción de esta variable se consideró desde la educación primaria hasta el posgrado.

- Dedicación. Es una variable discreta que indica el total de horas semanales que el empresario dedica a su actividad o negocio y que puede tomarse como un proxy de la actitud y ambición empresarial del propietario. Se consideró un mínimo de seis y un máximo de 145 horas semanales.

- Habilidades. Es una variable categórica que indica el nivel de habilidad o capacitación requerida para desempeñar su trabajo y que toma los siguientes valores: Alto nivel de capacitación $=1$; Capacitación a nivel técnico $=2$; Capacitación menor $=3$; No se requiere capacitación $=4$.

Características de las empresas:

- Ganancias. Variable continua que indica en miles de pesos mexicanos (corrientes) las ganancias reportadas por el empresario. Se puede tomar como un proxy del éxito de la empresa.

- Trabajo asalariado. Indica el número de trabajadores asalariados que laboran para la empresa (se excluye al propietario).

- Trabajo total. Indica el número total de trabajadores que labora para la empresa. Incluye los asalariados y los sin pago, pero excluye al propietario. Tanto esta variable como la anterior pueden ser consideradas aproximaciones al nivel de éxito de la empresa. 
- Salud. Es una variable dicotómica que indica si el empresario está inscrito en un instituto de salud pública o privada; toma el valor de 1 si él se encuentra inscrito en un instituto de salud público y de 0 si no es así. Se toma como un factor positivo del bienestar de los microempresarios.

- Vida. Variable que mide en ańos el tiempo que lleva el empresario al frente de la empresa. Puede ser tomado como una aproximación de la edad y de la estabilidad de la empresa.

- Gremio. Variable categórica binaria con valor igual a 1 si el empresario forma parte de una asociación gremial y de 0 si es de otra forma. Se considera como un proxy a la vinculación externa del negocio.

- Local. Variable dicotómica binaria con valor igual a 1 si el empresario tiene un local fijo para desarrollar su negocio y de 0 si no sucede así. Se toma como un proxy de localización.

- Sector. Variable categórica que indica el giro al que pertenece la actividad o negocio que ejecuta el microempresario; tiene los siguientes valores: manufactura $=1 ;$ comercio $=2$; construcción $=3$ y servicios $=4$.

- Capital. Variable continua que indica el capital per cápita (medido en miles de pesos) invertido en la empresa. Es decir, es el capital total dividido entre el número de trabajadores que laboran en la empresa, incluyendo al propietario.

La tabla 1 resume las variables y las describe brevemente.

Tabla 1. Descripción de variables

\begin{tabular}{ll}
\hline Variable & Descripción \\
\hline Edad & Edad de los empresarios expresada en años. \\
Experiencia & Años de experiencia del empresario. \\
Género & Variable que identifica si el empresario es hombre o mujer. \\
Migración & Variable de tipo indicador que identifica si el empresario cuenta con experiencia asalariada o no. \\
Escolaridad & Variable de tipo indicador que identifica si el empresario es migrante. \\
Dedicación & Número de años de educación formal del empresario. \\
Habilidades & Total de horas semanales que el empresario dedicó a su actividad o negocio. \\
Ganancias & Variable categórica donde se indica el nivel de habilidad requerido para su actual trabajo. \\
Trabajo asalariado & Ganancias reportadas por el empresario y provenientes de su actividad empresarial. \\
Trabajo total & Número de trabajadores asalariados que laboran para la empresa. \\
Salud & Número total de trabajadores que labora para la empresa. \\
Vida & Variable que identifica si el empresario está inscrito a algún instituto de salud pública o privada. \\
Gremio & Tiempo que tiene el empresario al frente de la empresa. \\
Local & Variable que identifica si el empresario está organizado gremialmente o no. \\
Sector & Variable que indica si el microempresario realiza su actividad en un local o no. \\
Capital & Variable categórica que clasifica la actividad que realiza el trabajador, como manufactura, \\
\hline & servicios, comercio o construcción. \\
& Capital por persona con la que cuenta la empresa medida en miles de pesos. \\
\hline
\end{tabular}

R. Rivera Huerta | Heterogeneidad en las microempresas informales mexicanas: evidencia empírica y algunas implicaciones de política | Perfiles Latinoamericanos, 26(51) | Flacso México | pp. 63-87 


\section{Resultados y análisis}

El análisis arroja cuatro categorías de empresas que se distribuyen según lo sintetiza la tabla 2 .

Tabla 2. Distribución de conglomerados (Porcentajes)

\begin{tabular}{lcc}
\hline \multicolumn{3}{c}{ Distribución de conglomerados } \\
\hline Clúster & Núm. & $(\%)$ \\
I & 382 & 5.7 \\
II & 2155 & 32.0 \\
III & 1951 & 29.0 \\
IV & 2238 & 33.3 \\
Total & 6726 & 100.0 \\
\hline
\end{tabular}

Fuente: Elaboración propia con datos de la Encuesta Nacional de Micronegocios (ENAMIN, 2008).

A continuación se presentan las características principales de cada uno de los clústeres (tablas 3 y 4 ).

Clúster I. Contiene al grupo de empresarios que es el más dinámico y el más pequeño (alrededor del $6 \%$ de la muestra). Es el clúster que reporta mayores ganancias (en promedio, alrededor de tres veces el clúster IV), el que tiene mayor capital invertido en las microempresas, el que dedica mayor tiempo a su negocio y el que emplea, en promedio, a mayor número de trabajadores pagados y trabajadores totales. A estos indicadores de "éxito" de los empresarios de este clúster se vinculan características generalmente entendidas como positivas, tanto de la empresa como del empresario: se organizan en gremios más que cualquier otro clúster, reportan el mayor porcentaje de empresarios inscritos en centros de salud públicos o privados, reportan más años de educación formal, dedican más tiempo a su actividad empresarial y, en promedio, es un clúster integrado por los empresarios más jóvenes. Es interesante destacar que la mayor parte de las empresas en este clúster se dedican a actividades sobre todo de servicios, aun cuando todos los sectores tienen, en alguna medida, presencia en él. Es pues un clúster comparable, a primera vista, a las mejores microempresas del sector formal, con abundancia considerable de capital humano y físico y capacidad para organizarse y vincularse. Este es, probablemente, el clúster que Fields denomina upper-tier, o a lo que en general se considera como una empresa "orientada al crecimiento".

Clúster II. Diversas características de los empresarios de este clúster permiten verlos como semejantes a los del I. Es el segundo con mayor capital invertido, 
aun cuando, en promedio, sea un tercio de lo que invierte el I y es el segundo con más empresarios inscritos en centros de salud pública o privada. Es también el segundo con mayor proporción de empresarios asociados en gremios. Sin embargo, es el de mayor edad, con mayor dedicación, y es la segunda categoría con más experiencia. Además, es el que tiene la menor proporción de trabajadores que reportan experiencia asalariada. No obstante, las variables que miden las ganancias y el número de trabajadores involucrados en sus empresas (totales y pagados) se encuentran colocadas en la tercera posición, todas por debajo del clúster III. Las actividades económicas de este conglomerado se concentran en el comercio, pero la manufactura y los servicios también participan de modo importante.

Clúster III. Este conglomerado es el más interesante porque ha escapado a los análisis previos de la literatura especializada. Destaca que una cantidad importante de las variables relacionadas con esta categoría señalan un desempeño positivo de sus empresas. Así, este es el clúster con el segundo nivel de ingreso (promedio) más alto y es el de los micronegocios más estables (años en vida de la empresa). Todo esto a pesar de que otras de sus variables no necesariamente son positivas, a saber: es el conglomerado con la segunda mayor edad promedio de los empresarios; el promedio de educación formal es el menor de todos los clústeres, y su capital invertido es aproximadamente el $40 \%$ del clúster II y el $10 \%$ del I. Finalmente, el porcentaje de empresarios asociados en gremios es extremadamente bajo y es el clúster con menor proporción de empresarios inscritos en centros de salud públicos o privados. Cabría preguntarse entonces, ¿cuáles son los factores subyacentes en esta dinámica relativamente exitosa? Los datos asociados a este clúster son los ańos de experiencia (la mayor, en promedio) y el tipo de experiencia (la mayoría reporta haber trabajado previamente como asalariado). Así pues, es posible pensar que el éxito relativo de las empresas que constituyen el clúster III se desprende de un aprendizaje no formal que se utiliza en la producción y servicios que brindan. Otro factor a considerar es el tipo de actividades en este clúster: $53 \%$ se concentran en la construcción y el resto en la manufactura. Por último, una tercera explicación la podrían constituir variables no medibles y, en consecuencia, no incorporadas en este trabajo, tales como las relativas a la psicología de los empresarios.

Clúster IV. Esta categoría la conforman microempresas cuyos rasgos se han asociado tradicionalmente con el sector informal. Es decir, son pequeñas actividades empresariales con poco capital invertido, que requieren pocas habilidades técnicas para su desempeño y que se encuentran enclavadas mayoritariamente en los sectores de comercio o de servicios. Los microempresarios propietarios de este clúster cuentan con el segundo nivel menor de educación promedio, aun 
cuando este sea muy similar al de las empresas del III. Adicionalmente, son empresarios con poca estabilidad promedio en sus actividades como tales. Reportan, asimismo, el menor número de trabajadores (sean pagados o de otra forma), lo cual da una idea de su pequeño tamaño. Es destacado que la técnica de conglomeración usada da como resultado que el 100\% de los empresarios de este grupo son mujeres. Por todo ello este conglomerado se asocia fácilmente al grupo de actividades que Fields denominó lower-tier, o con las empresas de "supervivencia".

Los resultados del análisis clúster son interesantes y enriquecen la discusión sobre la informalidad. En particular: a) los datos muestran que la dinámica de los empresarios (medida en ganancias) se relaciona claramente con las variables de educación formal, capital físico y dedicación empresarial. b) Los conglomerados parecen sugerir que no solo la educación formal desempeña un papel como capital humano en la dinámica de las empresas informales, de hecho se observa (véase el clúster III) que algunos empresarios podrían sustituir efectivamente la educación escolarizada con el conocimiento técnico adquirido a través de la experiencia. c) Es importante resaltar que el grupo de empresarios que, en promedio, perciben ganancias menores (clúster IV) es también el que reporta el menor porcentaje de los que han trabajado como asalariados. Cabría preguntarse si las características que marginan a los trabajadores en el clúster menos dinámico son las mismas que restringen su entrada al trabajo asalariado decente. $d$ ) Otras variables parecen estar jugando un papel en el desarrollo de las microempresas, entre ellas, el nivel de interacción con otros agentes económicos o sociales. Es notable cómo las categorías II y III son las que tienen menor proporción de empresarios asociados a gremios o con servicios de salud.

Los resultados de este ejercicio de análisis clúster de alguna forma respaldan la propuesta expresada en la introducción: las microempresas informales constituyen un grupo heterogéneo de actividades con diferentes potencialidades y carencias. Por tanto, dadas las diversas características de los grupos enmarcados como microempresas informales, las políticas públicas diseñadas para mitigar los problemas asociados a este sector deben ser también heterogéneas.

Así, el clúster I podría considerarse una agrupación de empresarios dinámicos y exitosos, cuya razón de permanencia en la informalidad se vincula a una decisión racional y a una preferencia por desempeñar actividades empresariales. Desde esta perspectiva, es factible que dichos empresarios consideren la informalidad como una ventaja que les permite evadir las obligaciones fiscales y de seguridad social impuestas por el Estado y, en consecuencia, observen dicho estatus como una ventaja competitiva. En contraste, este no sería el caso del clúster IV, el cual puede verse como un grupo de trabajadores marginados, que se encuentra en 
la informalidad debido a que quizá, por diversas causas, no pueden dedicarse a otra cosa, aunque deben cooperar con el gasto familiar.

La creación de políticas públicas para estos dos grupos debe seguir caminos diferentes. Parece que se puede atraer con relativa facilidad a los microempresarios del clúster I hacia la formalidad debido a que es probable que estos microempresarios tengan la capacidad de absorber los costos de la formalidad, siempre y cuando sean capaces de percibir sus beneficios. Entonces, la forma de atraerlos sería con la generación de políticas productivas destinadas a las microempresas formales dinámicas, mediante una simplificación administrativa que permita formalizarse fácilmente, así como de la instauración de políticas punitivas por evasión de sus obligaciones fiscales y laborales.

El caso del clúster IV es casi opuesto al del I: estos empresarios difícilmente podrían soportar cargas onerosas de impuestos y tendría poco sentido social imponérselas. Por ello, y coincidiendo parcialmente con Berner et al. (2012), las políticas dirigidas a este subsector serían: a) la creación de empleo formal que requiera poca educación o capacitación; b) creación, expansión y difusión de políticas de microcrédito especialmente dirigidos a este grupo; c) la creación de políticas sociales de género, $\mathrm{y} d$ ) cursos de capacitación básicos que les permitan gestionar mejor su negocio.

Más complicado es el caso de los clústeres de ingreso medio II y III. Sin embargo, los datos sugieren que las políticas de desarrollo dirigidas a incentivar su productividad tienen un papel más importante en comparación con los otros dos clústeres. En estos conglomerados, los empresarios registran un nivel de habilidad importante, adquirida mediante la educación formal (clúster II) o de la experiencia obtenida con su paso como asalariados en otras empresas (clúster III). Se observa, entonces, que la principal diferencia de estos conglomerados respecto del clúster I es el nivel del capital invertido. Por esto es factible que las políticas destinadas a otorgar créditos tendrían especial importancia para los empresarios de estos clústeres.

Sin embargo, las políticas de apoyo no deben limitarse a lo monetario. Obsérvese que, en especial, el clúster III reporta porcentajes extraordinariamente bajos de empresarios relacionados a asociaciones gremiales o a servicios de salud, lo cual repercute de modo negativo en el bienestar económico y social de sus propietarios. Es decir, se trata de empresas que se comportan de forma individualista y aislada. Fortalecer la cooperación productiva entre empresas con estatus de informalidad y vincularlas productivamente con sus contrapartes formales tendría profundos efectos en su integración con la sociedad y en estimular su bienestar social y su desarrollo económico. Además, relacionarlas con instituciones académicas incentiva la innovación, el aprendizaje y la difusión de conocimiento en ambos sentidos. 
Tabla 3. Características de los clústeres. Variables numéricas (medias)

\begin{tabular}{lcccc}
\hline & \multicolumn{1}{c}{ Clúster I } & Clúster II & Clúster III & Clúster IV \\
\hline Edad & Características de los emprendedores & & \\
Experiencia & 42.35 & 42.48 & 43.33 & 42.36 \\
Escolaridad & 25.62 & 26.71 & 28.42 & 23.35 \\
Dedicación & 12.17 & 9.70 & 7.72 & 7.77 \\
\hline \multicolumn{7}{l}{ Características de las empresas } & & & \\
\hline Ganancias & 16.39 & 5.18 & 5.37 & 2.55 \\
Vida & 12.20 & 11.47 & 13.99 & 8.49 \\
Capital & 99.82 & 21.84 & 11.32 & 6.32 \\
Trabajo asalariado & 1.88 & 0.11 & 0.24 & 0.06 \\
Trabajo total & 2.23 & 0.26 & 0.41 & 0.35 \\
\hline
\end{tabular}

Fuente: Elaboración propia con datos de la Encuesta Nacional de Micronegocios (ENAMIN, 2008).

Tabla 4. Características de los clústeres. Variables categóricas (porcentaje)

\begin{tabular}{|c|c|c|c|c|c|}
\hline & & Clúster I & Clúster II & Clúster III & Clúster IV \\
\hline \multicolumn{6}{|c|}{ Características de los empresarios } \\
\hline Experiencia asalariada & Ha trabajado como asalariado & 90.6 & 88.6 & 91.0 & 70.8 \\
\hline Migración & Nacido en esa ciudad & 64.9 & 65.7 & 68.9 & 67.0 \\
\hline Género & Hombre & 85.1 & 92.3 & 100.0 & 0.0 \\
\hline \multirow{4}{*}{ Habilidades } & Alto nivel & 29.3 & 13.5 & 0.0 & 0.0 \\
\hline & Nivel técnico & 21.5 & 15.7 & 0.7 & 0.0 \\
\hline & Capacitación menor & 39.5 & 23.3 & 99.3 & 59.7 \\
\hline & No se requiere & 9.7 & 47.4 & 0.0 & 40.3 \\
\hline \multicolumn{6}{|c|}{ Características de las empresas } \\
\hline Salud & Sí & 68.1 & 78.6 & 80.2 & 72.8 \\
\hline Gremio & Sí & 15.2 & 13.4 & 3.1 & 2.2 \\
\hline Local & Sí & 58.4 & 21.6 & 22.8 & 27.0 \\
\hline \multirow{4}{*}{ Sector } & Manufactura & 22.8 & 0.0 & 32.8 & 20.5 \\
\hline & Comercio & 13.4 & 14.5 & 18.5 & 44.4 \\
\hline & Construcción & 18.3 & 0.0 & 48.7 & 0.0 \\
\hline & Servicios & 45.5 & 85.5 & 0.0 & 35.1 \\
\hline
\end{tabular}

Fuente: Elaboración propia con datos de la Encuesta Nacional de Micronegocios (ENAMIN, 2008).

\section{Conclusiones}

A pesar de que es ampliamente aceptado que el conjunto de empresas con estatus de informalidad es altamente heterogéneo, pocos esfuerzos se han hecho para entender las particularidades de los subgrupos que lo conforman. Entender estas características se torna esencial para la creación, aplicación y evaluación de políticas públicas dirigidas a resolver, o cuando menos a reducir, los atributos negativos asociados a la informalidad, y a promover el desarrollo 
de sus potencialidades, a fin de mejorar el bienestar económico y social de los trabajadores en estos negocios. Elaborar una primera propuesta de clasificación que estimule la discusión sobre las políticas apropiadas para el desarrollo de estas empresas ha sido el principal objetivo de esta investigación.

Usando técnicas de análisis clúster y datos recopilados en 2008 por el INEGI mediante la ENAMIN, se ha explorado la heterogeneidad de las microempresas mexicanas con estatus de informalidad. Los datos identifican claramente a un subgrupo de empresas que reportan ingresos comparativamente altos, con abundancia relativa de capital humano y físico respecto de las otras categorías y que puede ser asociado con lo que otros estudios han etiquetado como microempresas orientadas al crecimiento. Las políticas de desarrollo para esta categoría podrían consistir en incentivos positivos para una incorporación a la formalidad (facilidad de acceso, créditos productivos) y negativos por pertenecer a esta, una estrategia basada en la racionalidad maximizadora de los agentes, propia de los modelos ortodoxos que abordan el tema del sector informal (Rivera-Huerta, 2014).

La evidencia muestra que la categoría orientada al crecimiento coexiste con otra que se caracteriza por una escasa dotación de capital humano y físico, una importante participación de las mujeres y un magro ingreso económico; todo lo cual conduce a que se la pueda equiparar con lo que la literatura especializada ha identificado como empresas de supervivencia. Para este tipo de negocios se requerirían — de modo parecido a lo que sugiere Berner et al. (2012) — políticas más cercanas a la asistencia social, créditos apropiados para empresarios a muy pequeña escala, pero, sobre todo, políticas a largo plazo enfocadas a un mayor crecimiento económico del país, mejor educación y creación de empleos que no requieran habilidades técnicas particulares.

Los resultados empíricos obtenidos en esta investigación respaldan la presencia contemporánea de empresas con un alto perfil frente a otras más modestas, tal y como las contribuciones teóricas usualmente reconocen. Como un producto adicional de esta investigación cabe observar la posible presencia de un segmento importante que no podría ser asociado de manera directa a alguno de los subgrupos de la dicotomía mencionada. Las empresas pertenecientes a este no perciben un ingreso que pudiera ser clasificado como alto, ni particularmente bajo, e insinúan un desempeño productivo aceptable. Resulta particularmente interesante el hecho de que esta dinámica económica no parece vincularse tanto con su nivel de capital, sino con su acervo de conocimiento: sus propietarios tienen altos niveles de capital humano adquirido a través de una educación formal o informalmente por medio de la experiencia. La existencia de este tipo de negocios indicaría que una clasificación de las microempresas informales en empresas orientadas al crecimiento y de supervivencia como suele registrarlo la literatura, no refleja la complejidad que los datos observados muestran. Aun cuando es necesario 
más trabajo para entender la naturaleza, potencialidades y necesidades de estas microempresas, el análisis aquí realizado indica que, aparte de un mayor acceso a capital físico y capacitación adecuada, ellas requieren incrementar su vínculo con el sector formal para fortalecer y mejorar su desempeńo.

Para concluir debe decirse que, en años recientes, el gobierno mexicano ha establecido una estrategia de políticas dirigidas a la formalización de las empresas, la cual se basa en la creación de estímulos productivos, simplificación administrativa y medidas punitivas que se orientan a los empresarios que evaden los reglamentos y las restricciones gubernamentales más básicas. Este es el caso del programa "Crezcamos Juntos" y otros de apoyo a la productividad de las microempresas, y del establecimiento casi paralelo de la reforma fiscal que incluye restricciones a los agentes económicos del comercio informal (Rivera-Huerta et al., 2016).

Las políticas del gobierno mexicano están claramente dirigidas a empresas orientadas al crecimiento; sin embargo, los datos señalan que estas últimas son una minoría. La evidencia que se ha expuesto en este artículo sugiere una participación en números absolutos significativamente mayor de microempresas de supervivencia. Si se considera que, como lo sostienen diversas fuentes, las empresas de supervivencia difícilmente evolucionan en categorías superiores, lo que se debe parcialmente a factores tales como los psicológicos-culturales, mismos que han sido hasta poco estudiados y comprendidos, las políticas implementadas serán poco eficientes desde el punto de vista económico y tendrán poco efecto a largo plazo en la reducción de la formalidad y en una mejora radical de la calidad de vida de los trabajadores.

Por otra parte, aun cuando el gobierno ha establecido programas de corte social como el Prospera que, por su naturaleza, se dirige a la población en pobreza, de la cual surge la mayor parte de los empresarios de supervivencia, los últimos reportes del CONEVAL (2015) indican que estos esfuerzos han sido insuficientes puesto que la población en pobreza ha aumentado. Tomando en cuenta estos datos, no habría motivo para esperar que disminuyan las empresas en informalidad en México. Y por ello es recomendable replantear la estrategia completa para el sector informal en México, comenzando por realizar estudios-estadísticos y de campo- que arrojen información sobre la naturaleza y las necesidades específicas que requiere cada subsector que integra el complejo mundo de las microempresas informales.

\section{Referencias}

Adams, A., Silva, J. de, Johansson, S. \& Razmara, S. (2013). Improving skills development in the informal sector. Strategies for Sub-Saharan Africa. Washington, D. C.: International Bank for Reconstruction and Development/The World Bank. 
Bangasser, P. (2000). The ILO and the informal sector: An institutional history. En ILo Employment Paper 2000/9 (pp. 1-64). Génova: International Labour Organization.

Baum, R. J., Locke, E. A. \& Smith, K. G. (2001). A multi-dimensional model of venture growth. Academy of Management Journal, (44), 292-302.

Berner, E., Gomez, G. \& Knorringa, P. (2012). Helping a large number of people become a little less poor: The logic of survival entrepreneurs. The European Journal of Development Research, 24(3), 382-396.

Capecchi, V. (1989). The Informal Economy and the Development of Flexible Specialization in Emilia Romagna. En Portes, A., Castells, M. \& Benton, L. (Eds.). The Informal Economy. Studies in Advanced and Less Developed Countries (pp. 189-215). Baltimore y Londres: The John Hopkins University Press.

Castells, M. \& Portes, A. (1989). World Underneath: The Origins, Dynamics and Effects of the Informal Economy. En Portes, A., Castells, M. \& Benton, L. (Eds.). The Informal Economy. Studies in Advanced and Less Developed Countries (pp. 11-37). Baltimore y Londres: The John Hopkins University Press.

Charmes, J. (1995). Una revisión crítica de los conceptos definiciones y estudios del sector informal. En Tokman, V. (Ed.). El sector informal en América Latina. Dos décadas de análisis (pp. 33-84). México: Consejo Nacional para la Cultura y las Artes.

Chen, M. A. (2012). The Informal Economy: Definitions, Theories and Policies. (wIEgo Working Paper núm. 1). Cambridge, M. A.: WIEGO.

Chen, M. (2007). Rethinking the Informal Economy: Linkages with the Formal Economy (DESA Working Paper núm. 46). Nueva York: United Nations.

Chen, M., Jhabvala, R. \& Frances, L. (2001). Supporting workers in the informal economy: A policy framework. Preparado para ILo Task Force on the Informal Economy. Cambridge, M. A.: WIEGO.

Chowdhury, H. (2005). Development the Concept of the Informal Sector: a Literature Review. Pakistan Journal of Social Sciences, 3(5), 724-732.

Coletto, D. (2010). The Informal Economy and Employment in Brazil. Latin America, Modernization, and Social Changes. Nueva York: Palgrave Macmillan.

CONEVAL (2015). CONEVAL informa los resultados de la medición depobreza 2014. Recuperado el 1 de junio de 2016, de http://www.coneval.org.mx/SalaPrensa/Documents/Comunicado005_ Medicion_pobreza_2014.pdf

R. Rivera Huerta | Heterogeneidad en las microempresas informales mexicanas: evidencia empírica y algunas implicaciones de política | Perfiles Latinoamericanos, 26(51) | Flacso México | pp. 63-87 
Cozzens, S. \& Sutz, J. (2014). Innovation in informal settings: Reflections and proposal for a research agenda. Innovation and Development (número especial), 4(1), 5-31.

Cunningham, W. \& William, M. (2001). Heterogeneity among Mexico's Microenterprises: An Application of Factor and Cluster Analysis. Economic Development and Cultural Change, 50(1), 131-156.

Dahl, M. \& Reichstein, T. (2007). Are You Experienced? Prior Experience and the Survival of New Organizations. Industry and Innovation, 14(5), 497-511.

Soto, H. de. (1989). The other path. Nueva York: Harper \& Row.

Fields, G. (2005). A Guide to Multisector Labor Market Models. The World Bank Social Protection, (0505), 1-50.

Fields, G. (2004). Dualism in the labor market: A perspective on the Lewis model after half a century. The Manchester School, 72(6), 724-735.

Freije, S. (2001). Informal Employment in Latin America and the Caribbean: Causes, Consequences and Policy Recommendations. Documento presentado en Regional Consultation on Labour Issues. Noviembre 26-27. Panamá: Instituto de Estudios Superiores de Administration (IESA).

Garoma, B. (2012). Determinants of Microenterprise Success in the Urban Informal Sector of Addis Ababa: A Multidimensional Analysis. Tesis doctoral. The Netherlands: Institute of Social Studies (ISS) - Erasmus University Rotterdam. Recuperado el 25 de junio de 2015, de repub.eur.nl/pub/37927/ThesisShaker\%5B1\%5D.pdf

Gërxhani, K. (2004). The Informal Sector in Developed and Less Developed Countries: A Literature Survey, Public Choice, 120(3/4), 267-300.

Goedhuys, M. \& Sleuwaegen, L. (2010). High-growth entrepreneurial firms in Africa: A quantile regression approach. Small Business Economics, 34(1), 31-51.

Hair, A., Anderson, R. E. \& Tatham, R. (1999). Análisis multivariante. Madrid: Pearson/Prentice Hall.

Harris, J. (1970). Migration, Unemployment and Development: A Two-Sector Analysis. The American Economic Review, 60(1), 126-142.

INEGi (2012). Encuesta Nacional de Micronegocios Enamin 2010 (documento metodológico). MéxiCO: INEGI. 
INEGI (2009). Encuesta Nacional de Micronegocios EnAmin 2008. México: INEgI.

INEGI (2004). La ocupación en el sector no estructurado en México. México: INEGI.

Jovanovic, B. (1982). Selection and evolution of industry. Econometrica, 50(3), 649-670.

Kirhlstrom, R. \& Laffont, J. (1979). A General Equilibrium Entrepreneurial Theory of Firm Formation Based on Risk Aversion. Journal of Political Economy, 87(4), 719-748.

Kriastensen, S. (2004). Social Networks and Business Success: The Role of Subcultures in an African Context. The American Journal of Economics and Sociology, 63(5), 1149-1171.

Kumar, H. \& Bhaduri, S. (2014). Jugaad to grassroot innovations: Understanding the landscape of the informal sector innovations in India. African Journal of Science, Technology, Innovation and Development, 6(1), 13-22.

Liedholm, C. (2002). Small Firm Dynamics: Evidence from Africa and Latin America. Small Business Economics, 18(1-3), 225-242.

Maloney, W. (2004). Informality Revisited. The World Development, 32(7), 1159-1178.

Maloney, W. (1999). Does Informality Imply Segmentation in Urban Labor Markets? Evidence from Sectoral Transitions in Mexico. The World Bank Economic Review, 13(2), 275-302.

McPherson, M. (1995). The Hazard of Small Firms in South Africa. The Journal of Development Studies, 32(1), 31-34.

Mead, D. \& Liedholm, C. (1998). The dynamics of micro and small enterprises in developing countries. World Development, 26(1), 61-74.

Nguimkeu, P. (2013). A Structural Econometric Analysis of the Informal Sector Heterogeneity. Journal of Development Economics, (107), 175-191.

Nguetse, P. (2009). Estimating the returns to education in Cameroon informal sector, en Scholar Materials and Research Al Tech (SMARTech). Recuperado el 30 de julio de 2015, de https:// smartech.gatech.edu/bitstream/handle/1853/34894/1244633932_PN_1.pdf

Organización Internacional del Trabajo (огт). (2013). La medición de la informalidad. Manual estadístico sobre el sector informal y el empleo informal. Turín, Italia: Organización Internacional del Trabajo-Centro Internacional de Formación de la oiт.

Pérez López, C. (2004). Técnicas de análisis multivariante de datos. Aplicaciones con SPSSS. Madrid: Pearson Educación.

R. Rivera Huerta | Heterogeneidad en las microempresas informales mexicanas: evidencia empírica y algunas implicaciones de política | Perfiles Latinoamericanos, 26(51) | Flacso México | pp. 63-87 
Rivera-Huerta, R. (2014). Rational Based Understanding of Informal Sector: A Critica Assessment. African Journal of Science, Technology, Innovation and Development, 6(3), 165-173.

Rivera-Huerta, R, López N. \& Mendoza A. (2016, enero-marzo). Políticas de apoyo a la productividad de la microempresa informal. ¿Dónde está México? Problemas del Desarrollo, 184(47), 87-109.

Romesburg, C. (2004). Cluster Analysis for Researchers, North Caroline: LULU PREsS.

Schneider, F. (2002). Further empirical results of the size of the shadow economy of 17 OECD countries over time. Discussion Paper Series Linz. Austria: Department of Economics, University of Linz.

Sinclair-Desgagné, B. (2013). Informal versus Formal New Venture: A Choice Analysis and Some Policy Implications. En Thai \& Turkina (Eds.). Entrepreneurship in the Informal Economy: Models, Approaches and Prospects for Economic Development (pp. 19-33). Nueva York y Londres: Routledge.

spss, Inc. (2001). The spss TwoStep Cluster Component. A scalable component enabling more efficient customer segmentation. White paper-technical report. Recuperado el 27 de agosto de 2017, de https://www.spss.ch/upload/1122644952_The\%20SPSS\%20TwoStep\%20 Cluster\%20Component.pdf

Swaminathan, M. (1991). Understanding the informal sector: A survey (wIDER Working Paper núm. 95). Cambridge, Massachusetts: World Institute for Development Economics Research of the United Nations University.

Tokman, V. (2011, septiembre-diciembre). Informalidad en América Latina: balance y perspectivas de políticas. Realidad, Datos y Espacio. Revista Internacional de Estadística y Geografia, 2(3), 16-31.

Tokman, V. (2001). De la informalidad a la modernidad. Oficina Internacional del Trabajo. En Integración de políticas. Boletín. OIT. Santiago: Oficina Internacional del Trabajo.

Tokman, V. (1995). Introducción: dos décadas del sector informal en América Latina. En Tokman, V. (Ed.). El sector informal en América Latina. Dos décadas de análisis (pp. 13-28). México: Consejo Nacional para la Cultura y las Artes.

Van Dijk, M. \& Fransen, J. (2008). Informality in Addis Ababa, Ethiopia. (Conferencia). En Are cities more important than countries? Rotterdam: Erasmus University. Recuperado el 1 de junio de 2015, de http://www2.econ.uu.nl/users/marrewijk/pdf/ihs\%20workshop/ fransen\%20paper.pdf 
Welter, F. (2001). Who wants to grow up? - growth intentions and growth profiles of (nascent) entrepreneurs in Germany. Presentado en Babson College-Kauffman Foundation Entrepreneurship Research Conference, Junio 14-16, 2001, Jönköping. Recuperado el 30 de julio de 2015, de https://www.researchgate.net/publication/38173322

Recibido el 19 de agosto de 2015. Aceptado el 9 de noviembre de 2016. 